\title{
Distribución de cargas en puentes vehiculares para su determinación de fallas
}

\section{Load distribution on vehicular bridges for failure determination}

\author{
DOI: $10.46932 / \mathrm{sfjdv3n1-095}$
}

Received in: Jan 30st, 2021

Accepted in: Feb 1th, 2022

\author{
Ramiro Chávez V \\ Departamento de Ingeniería Civil; Facultad de Ingeniería, \\ Universidad Autónoma de Querétaro, Santiago de Querétaro, 76010, México. \\ E-mail: rchavez02@alumnos.uaq.mx \\ G.J Ríos-Moreno \\ Facultad de Ingeniería, \\ Universidad Autónoma de Querétaro, Santiago de Querétaro, 76010, México. \\ E-mail: riosg@uaq.mx \\ M. Trejo-Perea \\ Facultad de Ingeniería, \\ Universidad Autónoma de Querétaro, Santiago de Querétaro, 76010, México. \\ E-mail: mtp@uaq.mx
}

\section{RESUMEN}

La mala distribución de cargas permanentes hacia los puentes y de su vida móvil, han provocado que surjan colapsos; los cálculos estructurales de cada puente son importantes y esto dependen de las cargas vivas y muertas, para que estos no lleguen a un colapso. El propósito de esta investigación es analizar la distribución de cargas en puentes para su determinación de fallas mediante datos estadísticos, con ello tener en cuenta las normativas para que los usuarios (empresas de carga) tengan en cuenta lo máximo que estos puedan soportar. Ensayos que se han realizado para diagnosticar las condiciones en las que se identifican daños graves respecto a las cargas que ejercen los vehículos y así tener en cuenta que tan seguros son después del diagnóstico, llevando así una presentación del puente en el software de AutoCAD. Los resultados muestran que las cuestiones climatológicas y cargas vehiculares a las que se encuentran constantemente los puentes, son factores que determinan el agrietamiento y su durabilidad en la vida útil.

Palabras clave: Cargas permanentes, cálculos en cargas, cargas de vehículo, puentes vehiculares.

\begin{abstract}
ABSTRAC
The poor distribution of permanent loads towards the bridges and their mobile life, have caused collapses to arise; the structural calculations of each bridge are important and this depends on the live and dead loads, so that they do not collapse. The purpose of this research is to analyze the distribution of loads in bridges to determine failures through statistical data, thereby taking into account the regulations so that users (freight companies) take into account the maximum that these can withstand. Tests that have been carried out to diagnose the conditions in which serious damage is identified with respect to the loads exerted by the vehicles and thus take into account how safe they are after the diagnosis, thus leading to a presentation of the bridge in the AutoCAD software. The results show that the weather issues and
\end{abstract}


vehicular loads that bridges are constantly faced with are factors that determine cracking and their durability over the useful life.

Keywords: Permanent loads, load calculations, vehicle loads.

\section{INTRODUCCIÓN}

Uno de los factores más importantes de la infraestructura internacional y nacional sin lugar a duda son los puentes vehiculares, ya que estos nos permiten salvar de un accidente geográfico como lo pude ser en un rio, un cañón, un valle o un cuerpo de agua, o cualquier otro obstáculo físico, como una carretera, un camino, una vía férrea, estos se diseñan de acuerdo a lo que se requiera y también al cómo se encuentre el área o terreno sobre el que se va a construir el puente.

Al realizar el diseño de la estructura del puente, esta debe ser capaz de cumplir con las funciones a la que se pretenda utilizar. De esta forma, nos muestra que todo puente siempre será susceptible de un deterioro gradual y acumulativo a lo largo de su etapa de servicio, a tal punto que el seguimiento, reparación y restauración de su estructura son uno de los principales retos de la obra civil (Manual de Inspección de Puentes, 2018).

De esta forma, los puentes, por sus condiciones de servicio, están constantemente sometidos a ciclos de carga y descarga debido al paso de vehículos que pasan por ellos, de esta manera los hace susceptibles al deterioro por fatiga, fenómeno que se presenta como un cambio micro estructural gradual y permanente en los materiales debido a la aplicación constante de cargas. Es sumamente importante ya que radica en el hecho de que, aunque la tensión nominal está por debajo del límite elástico del material, las tensiones locales cerca del final de una falla pueden ser lo suficientemente altas como para provocar su propagación, provocando eventualmente que la estructura alcance su colapso (Hillebrand, 2020). De los diferentes tipos de infraestructura civil, en el pasado reciente han enfatizado la necesidad y evaluación de puentes (Ahmed, 2020), el monitoreo de la infraestructura pública es importante, ya que es posible actuar con anticipación para que no exista falla alguna y que provoque daños a la población civil, ya que puede haber un colapso puesto que estas infraestructuras se utilizan a diario.

El agrietamiento en las vigas concreto pre forzado suele deberse a diversas causas, ya que durante su período de servicio o incluso durante su fabricación, se ha tenido en cuenta que en algunas vigas de este material ya se han formado fisuras previas a la liberación de la fuerza pretensora, estos parten del extremo superior y se extienden hacia el borde inferior, llegando incluso a penetrar en varios casos a lo largo de todo el peralte (Wang, 2020). Por otro lado, también se puede desarrollar agrietamiento por flexión, formada por sobrecargas e incluso por cargas de servicio regulares, considerando que puede generarse por defectos de fatiga si los elementos estructurales soportan cargas que producen tensiones de 
tracción en el hormigón (Hegger, 2020), que es el límite de tensión en la fibra extrema de concreto pre comprimido que permite la normativa norteamericana para el diseño de puentes, elaborada por la American Association of State Highway and Transportation Official (AASHTO, 2017).

Por su parte, también se ha abordado ampliamente el agrietamiento del concreto por efectos de fatiga (Gao, 2020). Sus modelos de crecimiento de grietas se han utilizado para predecir el deterioro por fatiga por efectos del tráfico en puentes (Manual de Inspección de Puentes, 2018), sin embargo, solo han sido para aquellos construidos con concreto reforzado, cuya ausencia de fuerza de pres fuerzo provoca un comportamiento y mecanismos de deterioro diferentes a las que se dan en elementos de concreto pres forzado, esto debido a las mismas circunstancias de los errores de cálculo que se realizan, pero la mayoría de las veces el factor de los componentes climatológicos como actor principal de las causas de las fisuras. El propósito de esta investigación es analizar la distribución de cargas en puentes para su determinación de fallas mediante datos estadísticos, con ello tener en cuenta las normativas para que los usuarios (empresas de carga) tengan en cuenta lo máximo que estos puedan soportar y tener una inspección adecuada previo a la elaboración del puente. El monitoreo hacia los puentes, es parte fundamental para que de alguna forma no se presenten problemas lo largo del tiempo, de la misma manera se verán reflejados muchos factores para el enriquecimiento para nuevos modelos y tener en cuenta las fallas que se presentan con más frecuencia en los puentes.

\section{CONSIDERACIONES TEÓRICAS}

Para llevar a cabo todo tipo de construcciones, es necesario ceñirse a las normas de la ley, como la normativa, ya que de esta manera existen establecimientos de construcciones más grandes y de tal manera que nos permite no cometer errores a lo largo de la misma. Es por ello que al realizar una obra existen ciertas restricciones laborales, en esta ocasión nos regiremos por la NOM-086-SCT2-2015 Oficial Mexicana, dispositivos de señalización y protección en áreas de obras viales (NOM, 2015), de esta manera, se conocerán las normas que se requerirán para el desarrollo de un puente vialidad, también es necesario establecer los requisitos generales que deben cumplirse para diseñar e implementar los dispositivos de señalización y protección en las áreas de obras viales de las carreteras y vías urbanas bajo jurisdicción federal, estatal y municipal, a fin de que dicha marcación sea uniforme en todo el territorio nacional con el fin de facilitar a los usuarios la comprensión de las indicaciones que transmite en relación a su seguridad y para reducir la ocurrencia de accidentes.

En cuanto a la realización de todo tipo de procesos con base a los puentes, se tiene que realizar un reconocimiento físico que se realiza principalmente a través de la vista, esta inspección se define como el conjunto de actuaciones técnicas realizadas según un plan previo que aportan los datos necesarios para 
conocer el estado físico de la estructura de un puente, la recopilación de información incluirá (en caso de ser posible): historia del puente, expedientes técnicos del proyecto, planos post-construcción, inspecciones previas, etc., hasta la salida a campo. Normalmente cada estándar o manual tiene que ser actualizado constantemente por cuestiones de nuevos materiales y cuestiones tecnológicas, por eso existen herramientas informáticas, ya que es una organización orientada a mantener el funcionamiento de los puentes que gestiona y aplica de forma óptima los recursos disponibles en beneficio de los usuarios, permitiendo así la gestión ágil y eficiente de la información.

Por estos motivos se debe tener en cuenta la manera de trayectoria que ejercen los vehículos, Ecuación (1):

$$
\begin{gathered}
{\left[M_{v}\right]\left\{\ddot{d}_{v}\right\}+\left[C_{v}\right]\left\{\dot{d}_{v}\right\}+\left[K_{v}\right]\left\{d_{v}\right\}} \\
=\left\{F_{G}\right\}+\left\{F_{v}\right\}
\end{gathered}
$$

donde $\left[M_{v}\right],\left[C_{v}\right]$ y $\left[K_{v}\right]=$ las matrices de masa, amortiguación y rapidez del vehículo, respectivamente; $\left\{d_{v}\right\}=$ el vector de desplazamiento del vehículo; $\left\{F_{G}\right\}=$ el vector de gravedad del vehículo; y $\left\{F_{v}\right\}=$ el vector de las fuerzas de interacción rueda-carretera que actúan sobre el vehículo (Deng, 2016).

La dinámica de un puente se puede describir como la siguiente Ecuación (2):

$$
\left[M_{b}\right]\left\{\ddot{d}_{b}\right\}+\left[C_{b}\right]\left\{\dot{d}_{b}\right\}+\left[K_{b}\right]\left\{d_{b}\right\}=\left\{F_{b}\right\}
$$

donde $\left[M_{b}\right],\left[C_{b}\right], \mathrm{y}\left[K_{b}\right]=$ masa del puente, amortiguación y rigidez matrices, respectivamente; $\left\{d_{b}\right\}=$ vector de desplazamiento del puente; y $\left\{F_{b}\right\}=$ el vector de las fuerzas de interacción ruedacarretera que se aplican al puente (Cal, 2016).

Las fuerzas de interacción entre el puente y el vehículo son fuerza y fuerzas de reacción que son iguales en magnitud, pero con direcciones opuestas. En los puntos de contacto, el desplazamiento del puente, el desplazamiento del eje de la rueda, la deformación del muelle del neumático y la rugosidad de la carretera son compatibles (Deng, 2016), Ecuación (3) 


$$
\begin{aligned}
& {\left[\begin{array}{ll}
M_{b} & \\
& M_{v}
\end{array}\right]\left\{\begin{array}{l}
\ddot{d}_{b} \\
\ddot{d}_{v}
\end{array}\right\}+} \\
& {\left[\begin{array}{ccc}
C_{b} & +C_{b b} & C_{b v} \\
C_{v b} & C_{v}
\end{array}\right]\left\{\begin{array}{l}
\dot{d}_{b} \\
\dot{d}_{v}
\end{array}\right\} 4+} \\
& {\left[\begin{array}{ccc}
K_{b} & +K_{b b} & K_{b v} \\
K_{v b} & K_{v}
\end{array}\right]\left\{\begin{array}{l}
d_{b} \\
d_{v}
\end{array}\right\}=\left\{\begin{array}{c}
F_{b r} \\
F_{v r}+F_{G}
\end{array}\right\}}
\end{aligned}
$$

donde $C_{b b}, C_{b v}, C_{v b}, K_{b v}, K_{v b}$ son los términos de amortiguación y rigidez relación la interacción entre el puente y el vehículo; $F_{b r}$ y $F_{v r}$ debido a la fuerza de contacto entre el puente y el vehículo y también son términos dependientes del tiempo (Deng, 2016).

Un perfil de la superficie de la carretera puede generalmente tratar como un proceso aleatorio gaussiano estacionario de media cero. Se puede generar un perfil de superficie de carretera aleatorio, Ecuación (4):

$$
\begin{gathered}
r(X)=\sum_{k=1}^{N} \sqrt{2 \varphi\left(n_{k}\right) \Delta n} \cos \left(2 \pi n_{k} X\right. \\
\left.+\theta_{k}\right)
\end{gathered}
$$

donde $\varphi\left(n_{0}\right)$ es la función ( $\mathrm{m}^{3} /$ cycle); $\theta_{k}$ es una función aleatoria uniformemente distribuida de 0 a $2 \pi ;$ y $n_{k}$ es el número de onda (ciclo/m) después de fue fundada otra operación, Ecuación (5)

$$
\varphi(n)=\varphi\left(n_{0}\right)\left(\frac{n}{n_{0}}\right)^{-2} \quad\left(n_{1}<n<\right.
$$

$$
\left.n_{2}\right)
$$

donde $n_{0}$ es la frecuencia de discontinuidad de $1 / 2 \pi($ cycle $/ m) ; \varphi\left(n_{0}\right)$ es el coeficiente $\left(\mathrm{m}^{3} /\right.$ cycle $)$ cuyo valor se elige en función del estado de la carretera; y $n_{1}$ y $n_{2}$ son los inferiores y superiores frecuentes al corte, respectivamente.

El deterioro progresivo bajo la acción combinada de cargas de tráfico y ambientales de corrosión, para describir su deterioro se utiliza la siguiente Ecuación (6)

$$
\begin{gathered}
\varphi\left(n_{0}\right)_{t}=6.1972 \times 10^{-9} \times \\
\exp \left\{\left[1.04 e^{\mathrm{n} t} * I R I_{0}+263(1+\right.\right. \\
\left.\left.S N C)^{-5}(C E S A L)_{t}\right] / 0.42808\right\}+2 \times 10^{-6}
\end{gathered}
$$


donde $\varphi\left(n_{0}\right)_{t}$ es el coeficiente de rugosidad de la carretera en función del tiempo; $I R I_{0}$ es el valor de rugosidad inicial antes de que la carretera se abra al tráfico; $\eta$ es el coeficiente ambiental que depende de las condiciones ambientales; t es el tiempo en años; $S N C$ es el número estructural calculado en función de la resistencia; $(C E S A L)_{t}$ es el número estimado de tráfico en términos de la carga por eje único (Bertola, 2019).

Los componentes del puente experimentarán ciclos de tensión complejos bajo la acción de pasar camiones, Ecuación (7)

$$
A F D(t)=\sum_{i} \frac{n_{i}}{N_{i}}
$$

donde $n_{i}$ es el número de ciclos acumulados en un nivel de rango de tensión de $S_{i}$ y $N_{i}$ es el número promedio de ciclos hasta fallas en esfuerzo. La fatiga especificando en las especificaciones de diseño de puentes AASHTO, $N_{i}$ y $S_{i}$ cumplen la siguiente Ecuación (8)

$$
N i=A S i m
$$

donde A es el coeficiente de resistencia a la fatiga $m$ es la constante de la pendiente. Después se sustituye la Ecuación (8) en la Ecuación (7), obteniéndose la Ecuación (9)

$$
\text { AFDt=iniSi3A }
$$

El estrés complejo en ciclos inducidos por cada paso de camión, el daño por fatiga acumulativo se puede calcular en función de la tensión primaria o máxima de rango y el número equivalente correspondiente de ciclos de tensión, Ecuación (10)

$$
\begin{gathered}
E N S C=n u m+\left(\frac{S_{r 1}}{M R S}\right)^{m}+ \\
\left(\frac{S_{r 2}}{M R S}\right)^{m}+\cdots+\left(\frac{S_{r i}}{M R S}\right)^{m}+\cdots\left(\frac{S_{r c u t}}{M R S}\right)^{m}
\end{gathered}
$$

donde $n u m=$ el número de rango de tensión máximo causado por cada paso de camiones; $S_{r 1}=$ $(1 \cdots$ cut $)=$ los rangos de tensión de orden superior; y $S_{r c u t}=$ el rango de tensión de corte. La constante de pendiente $m$ se utiliza el algoritmo de recuento de flujo de lluvia para extraer el número de estrés varía desde el historial de estrés (Cal, 2016). 
Por lo tanto, la distribución logarítmica normal (FD) debido a cada paso de camión bajo diferentes RSC, denotado por

$$
j(j=1,2,3,4,5) \text {, Ecuación (11) }
$$

$$
F D_{j}=E N S C_{j} * M S R_{J}^{3}
$$

los FD promedio de simulaciones bajo la acción de la fatiga a consideración de la velocidad a la que va cada vehículo (Cal, 2016).

\section{METODOLOGÍA}

En México se han desarrollado dos sistemas de gestión de puentes: el primero se denominó Sistemas de Puentes Mexicanos (SIPUMEX), su establecimiento estuvo a cargo de la Dirección General de Conservación Vial de la Secretaría de Comunicaciones y Transportes (Manual de Inspección de Puentes, 2018) para la administración de los puentes de la red federal libre de peaje la cual tiene a su cargo. SIPUMEX tiende a calificar los puentes (Figura 1) en una escala de 0 a 5 , con una calificación de 0 correspondiente a estructuras en perfecto estado que no requieren atención y una calificación de 5 a estructuras en estado crítico que requieren atención inmediata, puesto que se encuentra en una situación crítica, por ejemplo, el 5, después el 4 y así sucesivamente. Parte del personal de la Secretaría destaca la medición de la resistencia del concreto por procedimientos no destructivos, la determinación del hormigón mediante reactivos químicos: la cuantificación del avance de la corrosión mediante la medición de potenciales con una semi pila-galvánica, la auscultación de fallas por la técnica de impacto-eco y mediante la aplicación de pulsos ultrasónicos.

Figura 1. Evaluación de puentes.

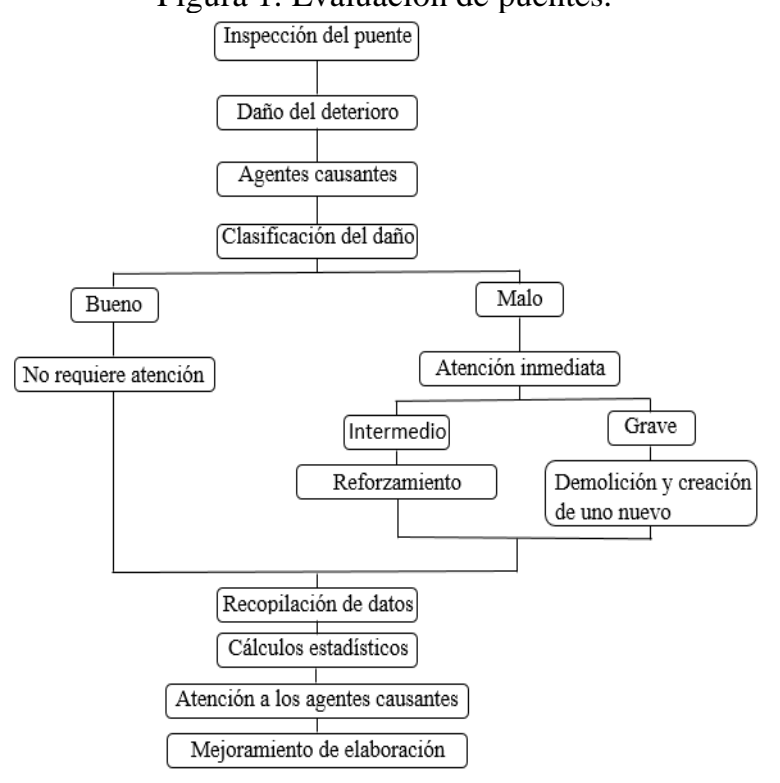


Como siguiente sistema, el Instituto Mexicano de Transporte desarrolló un sistema nacional llamado Sistema de Administración de Puentes (SIAP) que se ha aplicado en algunas autopistas concesionadas de cuota y también en la red a cargo del Organismo descentralizado caminos y Puentes Federales de Ingresos y Servicios Conexos (CAPUFE) (Manual de Inspección de Puentes, 2018). Estas entidades se han encargado de asegurar que las concesionarias implementen en la red a su cargo, un sistema de gestión de la conservación, que incluye un sistema de puentes. Las actividades que se han realizado han consistido principalmente en realizar un inventario y recopilar información para integrar el banco de datos. Es importante señalar que los puentes de la red de autopistas, por su modernidad, son en general más grandes y tienen estructuras más complejas que las de la red libre.

En su mayoría, los problemas que ocurren en los puentes, son causados por agentes naturales en los que la naturaleza intenta revertir el proceso artificial de fabricación de materiales de construcción y devolverlos a su estado original. El concreto (roca artificial) formado por agregados pétreos unidos con cemento y agua, sufre de agrietamiento y desconches, volviendo a convertirse en arena, grava y cemento separados, esto se debe a cambios de temperatura.

La aparición de vehículos cada vez más pesados en respuesta a la demanda de los transportistas que encuentran más lucrativa la operación de vehículos más pesados, explica la situación de daños en las estructuras de pavimentos y puentes, provocados por el aumento de tensiones, por el aumento del peso de las cargas rodantes y por la disminución de la resistencia por efecto de la fatiga estructural provocada por la frecuencia en la aplicación de estas cargas.

Se llevó a cabo una simulación de un puente en el software de AutoCAD donde se mostraron las partes importantes en donde a mayor frecuencia los defectos estructurales pueden ocurrir tales como: fisuras, grietas o fracturas en partes estructurales importantes como pilares y vigas, las fisuras por flexión son aquellas que generalmente se ubican en el área central del claro, incluyendo las áreas denominadas "momentos nulos", solo puede haber grietas inclinadas en la banda en las proximidades de los apoyos, sus grietas producidas por el estéreo cortante.

Las cargas están constituidas por los pesos propios de los materiales de todos los elementos que componen la superestructura, subestructura y cimentación, ya terminados. Incluye los pesos de: sistema de piso, elementos portantes, carpeta de calzada, parapetos, guarniciones, ductos, elementos complementarios, coronas y cuerpo de pilas o caballetes y el peso de zapatas, pilotes y cilindros o cajas de cimentación. En cuanto a los materiales que lo constituyen, pueden ser: concreto reforzado, concreto pres-forzado, acero estructural simple o pretensado y madera (Manual de inspección de puentes, 2018).

De cierta manera el proceso que se utiliza para un mayor conocimiento tiende a establecerse a las normas en las que lo especifican los regímenes gubernamentales, pues el principal dato que se debe tomar 
en cuenta es que la actual Norma Oficial Mexicana tiende al efecto, de la conservación de unidades en el Sistema Internacional (Tabla 1).

Tabla 1. Sistema Inglés (NOM, 2017).

\begin{tabular}{lccc}
\hline & Sistema Inglés & $\begin{array}{c}\text { Sistema } \\
\text { Unidades de Medida }\end{array}$ \\
\hline Peso & $1 \mathrm{lb}$ & 0,454 & $\mathrm{~kg}$ \\
Dimensiones & 1 pie & 0,3048 & $\mathrm{~m}$ \\
Potencia & 1 pulgada & 2,54 & $\mathrm{~cm}$ \\
$\begin{array}{l}\text { Momento de } \\
\text { fuerza }\end{array}$ & $1 \mathrm{HP}$ & 0,7457 & $\mathrm{Kw}$ \\
\hline
\end{tabular}

La finalidad de esta Norma es la clasificación de vehículos, según su clase (Tabla 2).

Tabla 2. Vehículo o Configuración Nomenclatura (NOM, 2017).

\begin{tabular}{cc}
\hline Clase: Vehículo o Configuración & Nomenclatura \\
\hline Autobús & $\mathrm{B}$ \\
Camión unitario & $\mathrm{C}$ \\
Tractocamión & $\mathrm{T}$ \\
Convertidor & $\mathrm{D}$ \\
Camión-remolque & $\mathrm{C}-\mathrm{R}$ \\
Tractocamión articulado & $\mathrm{T}-\mathrm{S}$ \\
Tractocamión doblemente articulado & T-S-R Y T-S-S \\
\hline
\end{tabular}

Teniendo en cuenta su clase, nomenclatura, número de ejes y neumáticos (Tabla 3).

Tabla 3. Características de los vehículos, en su nomenclatura, número de ejes y neumáticos, a) Autobús (B), b) Camión Unitario (C), c) Camión-remolque (C-R), d) Tractocamión articulado (T-S), e) Tractocamión semirremolque-remolque (T-S-R). Todos los camiones deben circular por carreteras y puentes de jurisdicción federal con luces encendidas permanentemente (NOM, 2017).

\begin{tabular}{|c|c|c|}
\hline Nomenclatura & Número de ejes & Número de llantas \\
\hline $\mathrm{B} 2$ & 2 & 6 \\
\hline B3 & 3 & $8-10$ \\
\hline B4 & 4 & 10 \\
\hline \multicolumn{3}{|l|}{ b) } \\
\hline Nomenclatura & Número de ejes & Número de llantas \\
\hline $\mathrm{C} 2$ & 2 & 6 \\
\hline $\mathrm{C} 3$ & 3 & $8-10$ \\
\hline \multicolumn{3}{|l|}{ c) } \\
\hline Nomenclatura & Número de ejes & Número de llantas \\
\hline $\mathrm{C} 2-\mathrm{R} 2$ & 4 & 14 \\
\hline C3-R2 & 5 & 18 \\
\hline C2-R3 & 5 & 18 \\
\hline C3-R3 & 6 & 22 \\
\hline
\end{tabular}


d)

\begin{tabular}{ccc}
\hline Nomenclatura & Número de ejes & Número de llantas \\
\hline T2-S1 & 3 & 10 \\
T2-S2 & 4 & 14 \\
& & \\
T2-S3 & 5 & 18 \\
& & \\
T3-S1 & 4 & 14 \\
& & \\
T3-S2 & 5 & 22 \\
& & \\
T3-S3 & 6 & \\
& & 22 \\
e) & & 22 \\
Nomenclatura & Número de ejes & Número de llantas \\
\hline T2-S1-R2 & 5 & 22 \\
T2-S2-R2 & 6 & 26 \\
T2-S1-R3 & 6 & 26 \\
T3-S1-R2 & 6 & 30 \\
T3-S1-R3 & 7 & 34 \\
T3-S2-R2 & 7 & 22 \\
T3-S2-R3 & 8 & 26 \\
T3-S2-R4 & 9 & 30 \\
T3-S2-S2 & 6 & \\
T3-S2-S2 & 7 & \\
T3-S3-S2 & 8 &
\end{tabular}

Las configuraciones T-S-R no incluirán semirremolques y / o remolques de tres ejes con eje retráctil, incluso cuando no se exceda el peso bruto máximo autorizado para estas configuraciones y el eje retráctil esté elevado. Las configuraciones vehiculares de tractor-semirremolque no pueden circular arrastrando un convertidor (Dolly) sin el semirremolque adjunto. Los autobuses, camiones unitarios (C) y tractores a partir de un peso bruto vehicular de $7.257 \mathrm{Kgs}$, deben tener un freno auxiliar. Los autobuses, los camiones unitarios (C), así como las configuraciones de camión con remolque (C-R) y articulada sencilla (T-S) deben tener un sistema de frenos antibloqueo. Los camiones unitarios, así como las configuraciones de camión-remolque (C-R) y articulada sencilla (T-S), deben tener cámaras de frenado de doble acción (estacionamiento y servicio) en todos los ejes, excepto el direccional, cuando están equipados. Los autobuses deben tener cámaras de frenado de doble acción (estacionamiento y servicio) en los ejes motrices, cuando estén equipados. Para las configuraciones de autobús, camión unitario y camión-remolque (C-R) y articulado simple (T-S), deben tener un sistema de ajuste automático de frenos. Las configuraciones camión-remolque (C-R) y tracto-camión articulado (T-S) deben tener espejos auxiliares en la parte delantera ubicados en las salpicaderas (guardabarros) y/o cubierta del motor, dependiendo del diseño de la carrocería o un elemento que permita la reducción de los puntos ciegos. Para vehículos con configuración de cabina sobre motor, pueden usar espejos auxiliares de acuerdo con el diseño del fabricante (NOM, 2017). 
La red vial nacional, su importancia y características, la red vial mexicana se clasifica en: red federal, redes estatales, caminos rurales y brechas mejoradas.

Puentes federales: En carreteras federales, el gobierno federal cuenta con todos los servicios.

Puentes estatales: Las redes estatales juegan un papel de gran relevancia para la comunicación regional, para vincular áreas de producción agropecuaria y para asegurar la integración de grandes áreas en diversas regiones del país.

Puentes municipales: Caminos rurales y los espacios mejorados, que son caminos modestos y generalmente sin pavimentar; su valor es más social que económico, ya que brindan acceso a pequeñas comunidades que de otro modo estarían aisladas.

Puentes concesionados: acto mediante el cual el Estado otorga a una persona el derecho a prestar un servicio público y a explotar bienes de dominio público, (NOM, 2017).

Esta ocasión se basará en un puente Paso Inferior Vehicular (PIV) cuya estructura se construye en un cruce de la carretera de referencia debajo de otra carretera y cuyas dimensiones están definidas por las características geométricas y rasante de ambas carreteras. Los materiales más habituales para su construcción son el concreto ya que son los más habituales y se componen de una combinación de cemento Portland, áridos pétreos, agua y aditivos para formar una mezcla moldeable que al fraguar forma un elemento rígido y resistente. Estos pueden ser reforzados con varillas de acero (concreto reforzado) o con torones o cables de acero a los que se induce una carga de tracción (concreto pres-forzado) antes (pretensado) o después (postensado) de colocar el elemento.

Para la demanda que se presenta por las cargas vehiculares a las que está sometido un puente, es necesario considerar múltiples variables, tales como: configuraciones del vehículo, condiciones de carga y pesos brutos del vehículo, por lo que se utiliza un método de simulación que relaciona todas las variables, obtener las distribuciones probabilísticas de las cargas que imprime el tráfico vehicular.

\section{RESULTADOS Y DISCUSIÓN}

El pasaje analizado nos ofreció de cierto modo una pequeña introducción de algunas normas y reglas las cuales son de ayuda en la ingeniería civil, pero en este caso se adentró más en las estructuras de los puentes de vialidad y en el aspecto de cómo se distribuye las cargas y algunos aspectos de cómo el material es sin duda uno de los principales factores de grietas o fisuras las cuales se presenta en los puentes, los resultados que arrojen las formulas, está se harán los cálculos y establecerá una función de su distribución estadística. Se tomó en cuenta para la estructura de puente con hormigón pres-forzado, ya que de cierta manera en su aplicación es más fácil y se evita que en ocasiones se tenga errores, como vista previa el siguiente modelo de un puente, donde las vigas son tipo I las cuales son elaboradas de fábrica 
esto con el fin de acelerar el término del puente, ya que si se hacen en el lugar donde se desea llevarlo a cabo se alargaría el proceso, de esta forma también es de ayuda para la reducción de gastos puesto que solo se ensambla en las columnas y tiende al uso de grúas para su armando más práctico.

Un aspecto importante el cual no se debe de dejar por ningún motivo, es la revisión de la estructura del puente, ya que este tiende a estar en la intemperie entonces tiende a presentar daños naturales sobre sí mismo, un factor que muchas veces se tiene presente a simple vista, es en la manera de como los puentes están diseñados, para soportar cargas de gran magnitud de acuerdo a su estructura hay unas de poca y de gran capacidad, entonces luego hay empresas que por más facilidad para ellas mandan unidades con cargas más grandes de las cuales ya están establecidas, entonces estas mismas hacen que los puentes sufran daños, en cambio si se lleva de manera adecuada su uso, este tendrá una vida más larga y establecerá confianza a los usuarios.

Las Figuras 2 y 3 muestran una vista inferior del puente, donde se puede apreciar como la calzada, parapetos, guarniciones, ductos, guarniciones, ductos, elementos complementarios, coronas y cuerpo de pilas o caballetes y el peso de zapatas.

Figura 2. Vista inferior.

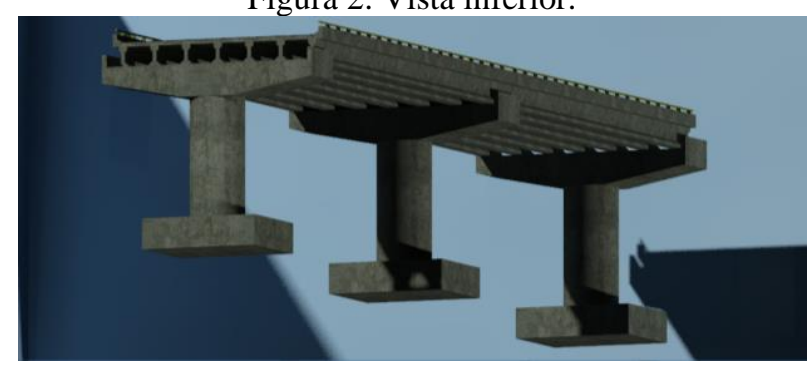

Figura 3. Vista de carril.

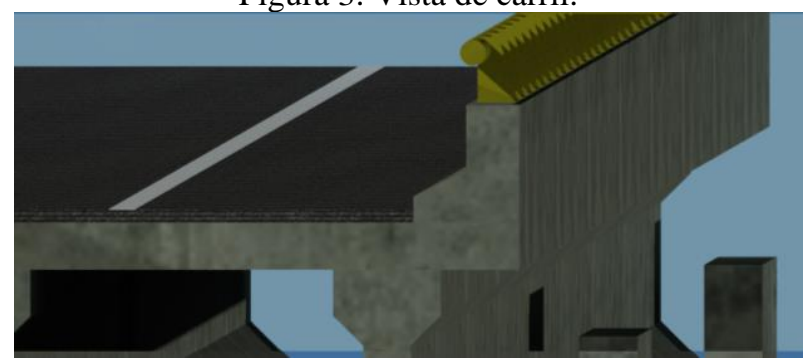

En la Figura 4 muestra los cilindros de cimentación, donde la viga de concreto pres-forzado.

Figura 4. Tamaño de viga de concreto pres-forzado tipo I.

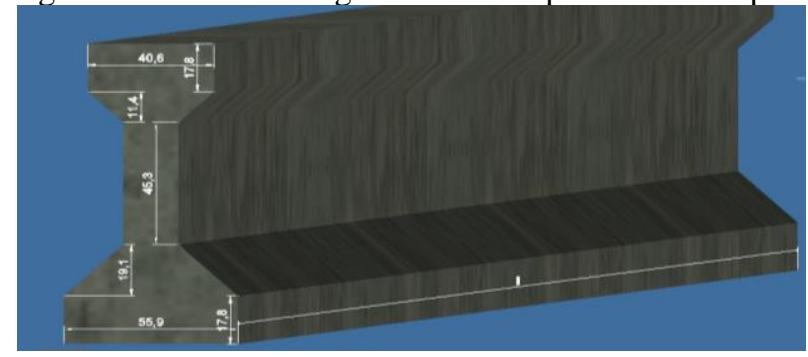


La Figura 5 indica una vista área del puente, mismo que se hizo de dos carriles con un mismo sentido, donde cuenta con un carril de baja y otro de alta velocidad. Cuando pasan dos vehículos al mismo tiempo, el de más peso deberá ir en el carril de baja y el más ligero en el de alta, sus dimensiones se muestran en la Figura 6.

Figura 5. Vista área.

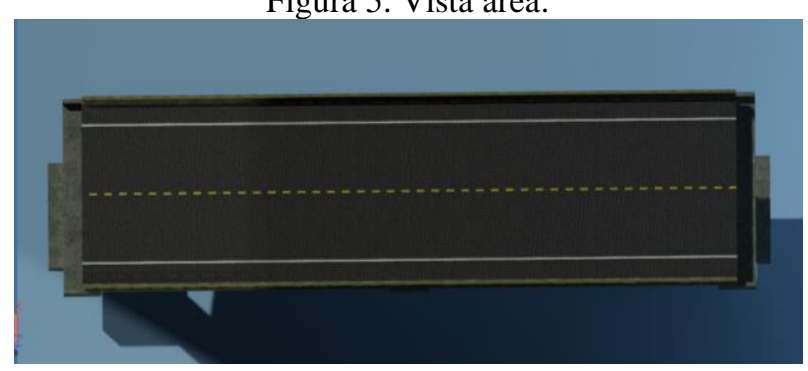

Figura 6. Las dimensiones de cada carril son de $3.65 \mathrm{~m}$, dejando un espacio del parapeto a una distancia de $1.10 \mathrm{~m}$. Cada viga está a una distancia de 1.55 tomando el centro del puente como referencia y son un total de 7 vigas.

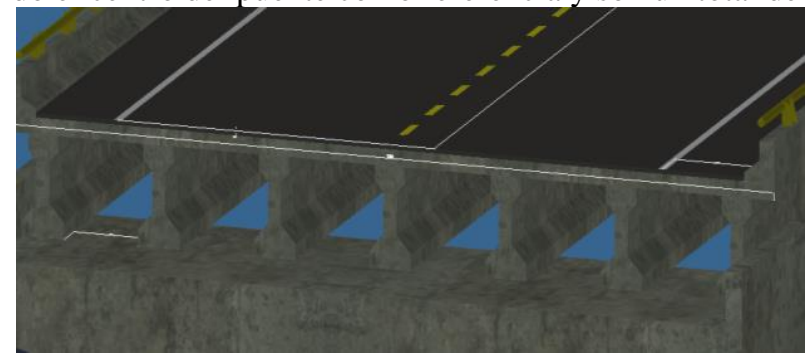

En vista lateral (Figura 7) el puente cuenta con cimientos o también conocidas como zapatas aisladas a una distancia de $22 \mathrm{~m}$, columnas a un peralte de $6 \mathrm{~m}$ a partir de la parte superior de la zapata, donde sus distribuciones de cargas son puntúeles, de acuerdo a este tipo de puente, tiene un parapete de protección para que algún vehículo salga disparado hacia los voladizos.

Figura 7. Vista lateral.

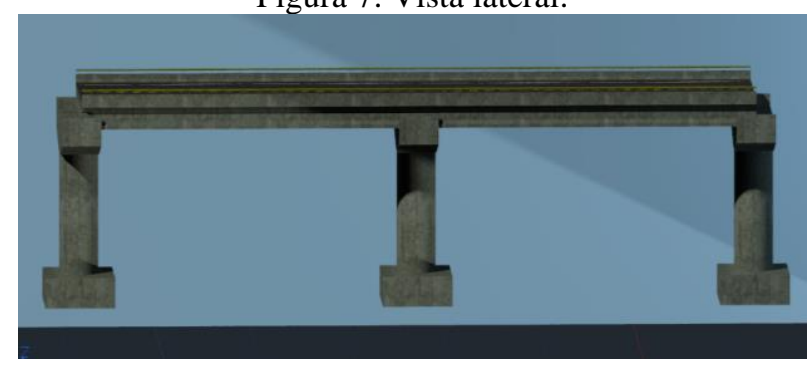

La mejora de puentes tanto en sus cálculos los cuales nos determinan donde hay agrietamiento y en citaciones se puede apreciar a simple vista y también en el tipo de material el cual debe estar construido, de esta forma los puentes tendrán una mejor vida y de la misma manera se generará confianza a los usuarios. 


\section{CONCLUSIONES}

El mejoramiento hacia los puentes es bajo las regulaciones requeridas en las cuales se mencionan en el Manual de Inspección de Puentes del 2018, que las cargas están constituidas por los pesos propios de los materiales donde todos los elementos forman la superestructura, subestructura y cimentación. En la NOM-086-SCT2-2015 Oficial Mexicana señala que a todo puente se debe realizar un reconocimiento físico realizándose a través de la vista donde será necesario que se recopilen datos, su historial donde se tenga una inspección posterior a la que se está haciendo, planos post-construcción y esto permite una gestión ágil. En SIPUMEX, el Sistema de Administración de Puentes (SIAP) desarrollado por el Instituto Mexicano de Transporte, tiende a calificar los puentes para poder saber si se necesita una reparación o no, teniendo en cuenta que se usa una escala del 0 a 5 , donde 0 corresponde a estructuras en perfecto estado y no requieren reparación alguna y 5 es donde se presenta la estructura en una situación crítica. Al realizar el diseño de la estructura del puente, esta debe ser capaz de cumplir con las funciones a la que se pretenda utilizar. De esta forma, nos muestra que todo puente siempre será susceptible de un deterioro gradual y acumulativo a lo largo de su etapa de servicio, a tal punto que el seguimiento, reparación y restauración de su estructura son uno de los principales retos de la obra civil. Teniéndose en cuenta el abuso hacia los puentes por parte de los usuarios que trasladan mayores cargas a las permitidas, el escaso monitoreo que se les brinda, cuestiones climáticas, consecuencias: el concreto (roca artificial) formado por agregados pétreos unidos con cemento y agua, sufre de agrietamiento y desconches, volviendo a convertirse en arena, grava y cemento separados. Mediante el recopilado de datos en el estudio, se facilitará la prevención de daños en los elementos estructurales y la aplicación de los regímenes establecidos, con la finalidad de brindar una infraestructura funcional. 


\section{REFERENCIAS}

Ahmed H. (2020). Revisión de la evaluación de infraestructura civil no destructiva para puentes: plataformas robóticas de última generación, sensores y algoritmos. Departamento de Informática e Ingeniería. Estados Unidos.

AASHTO (2017). AASHTO LRFD especificaciones de diseño de puentes. AportesIngeCivil. $8^{\text {a }}$ edición. Estados Unidos.

Bertola N.J. (2019). Una metodología para el diseño de sistemas de medición que combina información de excitaciones estáticas y dinámicas para pruebas de carga de puentes. Sonido. Estados Unidos.

Cal C.S. (2016). Efecto del ciclo de mantenimiento del pavimento sobre la confiabilidad a la fatiga de los puentes de vigas en I de acero con soporte simple bajo carga dinámica de vehículos. Dpto. De Ingeniería Civil y Ambiental. Estados Unidos.

Deng L. (2016). Efecto del ciclo de mantenimiento del pavimento sobre la confiabilidad a la fatiga de los puentes de vigas en I de acero con soporte simple bajo carga dinámica de vehículos. Laboratorio clave de ingeniería eólica y de puentes de la provincia de Hunan. China.

Gao Q. (2020). Un método basado en filtros de Kalman para diagnosticar la condición estructural de puentes de haz de luz media y pequeña durante breves interrupciones de tráfico. Escuela de Ciencias e Ingeniería del Transporte. China.

Hegger J. (2020). Ensayos de fatiga de refuerzo de cortante en vigas en T de hormigón pretensado de puentes. Instituto de Hormigón Estructural. Alemania.

Hillebrand M. (2020). Ensayos de fatiga de refuerzo de cortante en vigas en T de hormigón pretensado de puentes. Instituto de Hormigón Estructural. Alemania.

Manual de inspección de puentes (2018). Secretaria de Comunicaciones y Transportes. Dirección General de Servicios Técnicos. México.

NOM (2015). NORMA Oficial Mexicana NOM-086-SCT2. Señalamiento y dispositivos para protección en zonas de obras viales. México.

NOM (2017). NORMA Oficial Mexicana NOM-0120-SCT-2. Sobre el peso y dimensiones máximas con las que podrán circular los vehículos de transporte automotor que transiten en las vías generales de comunicación de jurisdicción federal. México.

Wang X. (2020). Un método basado en filtros de Kalman para diagnosticar la condición estructural de puentes de haz de luz media y pequeña durante breves interrupciones de tráfico. Instituto de Investigación Científica del Puente Ferroviario de China. China. 